\title{
DEDICATION
}

\section{Honouring the Life and Contributions of Liz Elliott}

T is with profound sadness that we inform you of the passing of Professor

Liz Elliott of Simon Fraser University's Institute of Restorative Justice and School of Criminology. Liz passed away peacefully, at home, in the company of family on 9 September 2011. She was an active member of the $J P P$ Editorial Board in the formative years of the Journal and she remained so until her passing. Her contributions - including as co-editor of volumes 5(2) with Gayle Horii, 9(1) with Stephen Reid and Peter Murphy, and 12 with Jay Jones, as well as serving in various other roles such as Editor have helped to sustain and grow the JPP.

Many of the JPP's contributors and readers first came into contact with the Journal through Liz's work. She was a passionate advocate for prisoners' rights, as well as aboriginal, restorative and social justice. Liz took up these causes in as many spheres as possible - as an educator, an advocate inside and outside of carceral spaces, and respected voice in public forums. Her work in British Columbia's prisons and in academia continued even as her health deteriorated, culminating in the publication of a book entitled Security with Care: Restorative Justice \& Healthy Societies (2011, Fernwood Publishing).

In some of our final correspondences she spoke of the urgency she felt when working with prisoners to facilitate healing and release in a carceral context increasingly characterized by "accumulating, blatant human rights abuses", overcrowding, and a pervasive and crippling drug culture. This issue of the JPP is dedicated to the memory of Liz Elliott: colleague, friend, mentor, fellow traveler and inspiration. 